\title{
Fast Quantification of Abdominal Aortic Aneurysms from CTA Volumes
}

\author{
O. Wink, W..J. Niessen, M.A. Viergever \\ Image Sciences Institute, Room E 01.334, \\ University Hospital Utrecht, Heidelberglaan 100, \\ 3584 CX Utrecht, the Netherlands \\ \{onno,wiro,max\}@isi.uu.nl \\ http://ww.isi.uu.nl
}

\begin{abstract}
A method is presented which aids the clinician in obtaining quantitative measurements of an abdominal aortic aneurysm from a CTA volume. These measurements are nceded in the preoperative evaluation of candidates for minimally invasive aneurysmal repair. The user initializes starting points in the iliac artery. Subsequently, an iterative tracking procedure outlines the central lumen line in the aorta and the iliac arteries. Quantitative measurements on vessel morphology are performed in the planes perpendicular to the vessel axis. The entire process is performed in less than one minute on a standard workstation. In addition to the presentation of the calculated measures, a 3D view of the vessels is generated. This allows for interactive inspection of the vasculature and the tortuosity of the vessels.
\end{abstract}

\section{Introduction}

An abdominal aortic aneurysm (AAA) is a life threatening dilation of the main artery, occurring mainly in men at older age. Standard treatment of an AAA involves the replacement of the dilated part of the aneurysm by a prosthesis. This invasive procedure has a postoperative mortality rate of about $7 \%$. Recently a less invasive, alternative treatment with potentially lower risk has emerged, which implies the introduction of a folded aortic graft through the femoral artery, using a groin incision. The dimensioning of grafts for this endovascular intervention is a key issue in the effort to minimize peri- and postoperative complications. The most common complications of inaccurate graft sizing are persistent endoleak and thrombosis of the graft body or limbs. In order to minimize inaccuracy in graft sizing, diameter measurements should be performed in planes perpendicular to the central vessel axis [1]. This central lumen line, the very basis for the dimensioning of the grafts, is currently drawn by the clinician, using three orthogonal views. The entire dimensioning process is very labor intensive, user dependent, and takes roughly one hour per patient.

The purpose of this study is to develop a fast objective method for the detection of the central lumen line, and to subsequently determine different quantitative measures along this line, in order to facilitate the preoperative evaluation of candidates scheduled for endovascular repair. 


\section{Related work}

Most methods of 3D line detection [2-4], are based on a two phase process. First a feature image is constructed that yields high responses in the center of the lumen. Second, a search process is started that tries to connect the different maxima from the preceding stage into a connected structure. These methods are computationally intensive, and exhibit varying results, especially in highly curved regions, and at the bifurcations.

In contrast, the homing cursor [5] directly explores the 3D volume, but assumes smoothly varying contours, and a Gaussian cross section. Because of the large variations in width of the vessels, and the presence of calcifications, this method is not suited for our application.

Other 3D applications [6-8] that are able to cope with the varying width of the vessel structure either use different preprocessing steps, like segmentation and/or manual delineation of the central lumen, before the actual quantification process takes place. Fiebich et al. [9] report successful automated segmentation of the aorta and its branching vessels, based on two different thresholds. However, the more difficult problem of segmenting the iliac and femoral arteries is not solved by this procedure. The variation in contrast of the iliac and femoral arteries is too large for a single threshold to yield a successful segmentation.

The method presented here, directly explores the 3D data. As a result, no segmentation is needed before the method can be applied. User interaction is minimal, only two starting points have to be indicated. Furthermore, the method can be used interactively.

\section{Current practice}

In current clinical practice at our hospital, the operator manually determines the central lumen line on a Philips EasyVision workstation, using three orthogonal views. Based on this line, and its perpendicular planes, it is determined whether the patient is suited for endovascular repair. If the iliac trajectory for example has a diameter less than $5 \mathrm{~mm}$, the graft may not be able to pass. The different measurements result in the dimensions of the graft and the preferred entry side (left or right), through which the graft will be introduced. In order to get an overview of the vasculature and the tortuosity of the iliac trajectory, the aorta is segmented manually. The segmentation is performed in the $2 \mathrm{D}$ slices, using basic thresholding and region growing techniques.

\section{Description of the method}

We propose a near automatic method for the detection of the central lumen line of the aorta and the iliac and femoral arteries. After the initial points have been determined by the operator, the tracking process is started. In every iteration the central lumen line is extended by one point. At this new position, a plane perpendicular to the central lumen line is constructed. This plane is subsequently 
searched for the center of the lumen, which results in an update of the central lumen line. During the process, the central lumen line and a corresponding confidence level are presented. This enables the user to monitor the process, and take over control if needed. In the next sections the different phases will be described in detail. First, the most important step, determining the lumen center, is discussed.

\subsection{Determination of the lumen center}

Given a plane, perpendicular to the central lumen line, we aim to compute the center of the lumen. Figure 1 gives three examples of such a cutplane. These
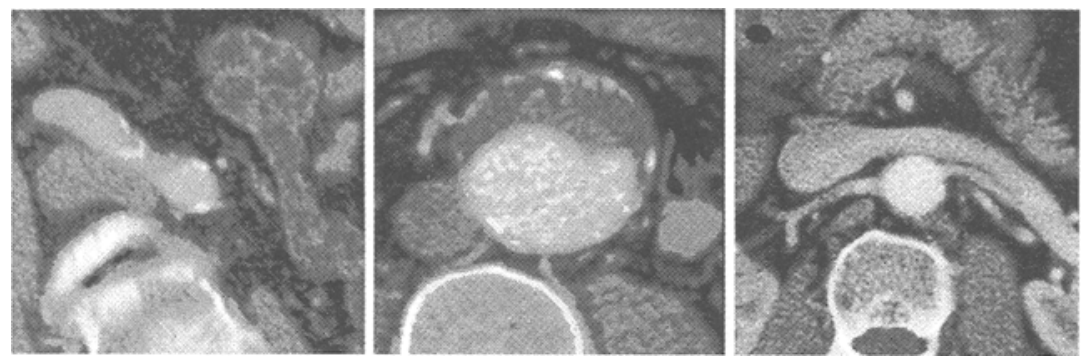

Fig. 1. Three examples of a perpendicular plane, in which the center of the lumen must be determined. In the left image, two iliac arteries, just below the bifurcation are visible. In the middle image the aneurysm itself is displayed. The right image shows the neck of the aneurysm and two renal arteries.

examples illustrate some specific problems that may be encountered in determining the center of the lumen. First, the calcifications appear as very dense spots, yielding a high response to any gradient integration based method. Second, both shape and size of the contour of the lumen are very unpredictable. This hampers template based or model based methods, and methods based on a fixed scale. Third, it may occur that the cutplane is almost parallel to one of the joining arteries, or is positioned at a bifurcation. Fourth, the intensity of the pixels in the contrast filled lumen may vary considerably.

We propose a method which is capable of coping with the problems described above. A schematic example of the method is given in figure 2. From the point

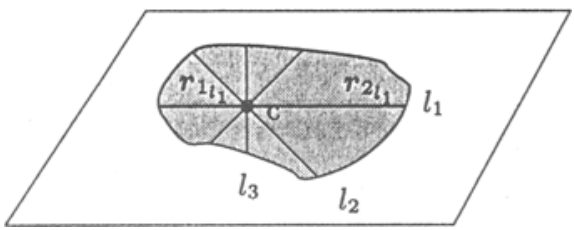

Fig. 2. Determination of the lumen center. From the point $c$, a number of rays is cast. For line $l_{1}$ there exists a pair of rays $\left(r_{1_{l_{1}}}, r_{2_{l_{1}}}\right)$. 
$c$ in the perpendicular plane, a number of 'rays' is cast. Every ray $r$ stops at the first gradient maximum above a threshold $t$. The gradient $\nabla L$ at scale $\sigma$ is computed using a convolution of the original image with a Gaussian derivative in the direction of the ray:

$$
\frac{\partial L}{\partial \boldsymbol{r}}=\frac{|\boldsymbol{r} \cdot \nabla L|}{|\boldsymbol{r}|}
$$

For every pair of rays $\left(\boldsymbol{r}_{1_{t_{i}}}, \boldsymbol{r}_{2_{t_{i}}}\right)$ along every line $i$, the length is determined. The confidence level $C L$ of a point is determined using:

$$
C L=\frac{1}{N} \sum_{i=1}^{N} \frac{\min \left(\left|\boldsymbol{r}_{1_{i_{i}}}\right|,\left|\boldsymbol{r}_{2_{l_{i}}}\right|\right)}{\max \left(\left|\boldsymbol{r}_{1_{i_{i}}}\right|,\left|\boldsymbol{r}_{2_{l_{i}}}\right|\right)}
$$

where $N$ is the number of lines. When the confidence level is computed for every point in a $30 \times 30$ grid around the center of the leftmost cutplane in figure 1 , we get the surface as shown in figure 3 . The point with the highest confidence
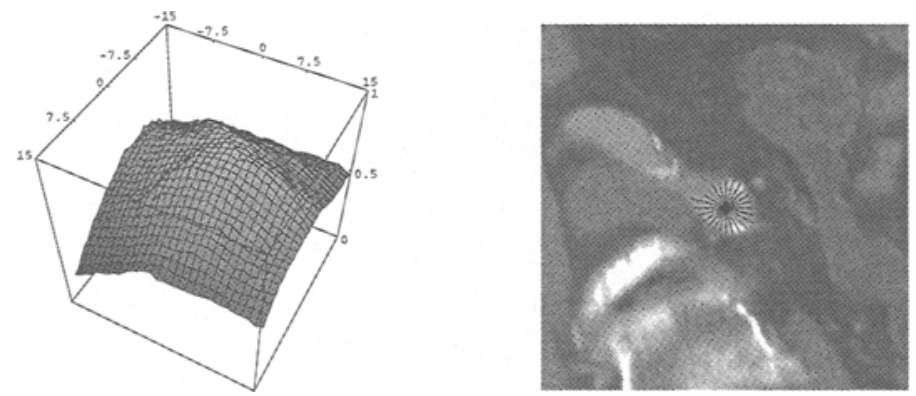

Fig. 3. Confidence level surface, and the rays used in computing the maximum confidence level, $\sigma=3, t=4, N=12$.

level is assumed to be the center of the lumen. The rays used to compute this maximum, are displayed in the right image of figure 3 .

The confidence level has several interesting properties, Some of these will now be discussed.

Calcifications: because the gradient is computed in the direction of the ray, the calcification is treated as being part of the lumen (sce the right image in figure 3). If only the gradient magnitude would be used, the ray would have stopped at the first boundary of the calcification it detects, thus giving an underestimate of the lumen width, and a possible error in the determined lumen center.

If a significant amount of calcium is present in one of the perpendicular planes, the method might find the center of a calcification instead of the center of the lumen. Therefore, we put a constraint on the minimum length of the lines. If the length of a line is below this threshold, the contribution to the confidence level will be zero. 
Bifurcations: if the cutplane is positioned at a bifurcation, the confidence level is still able to find the center. In figure 4 an example of a bifurcation is given. In
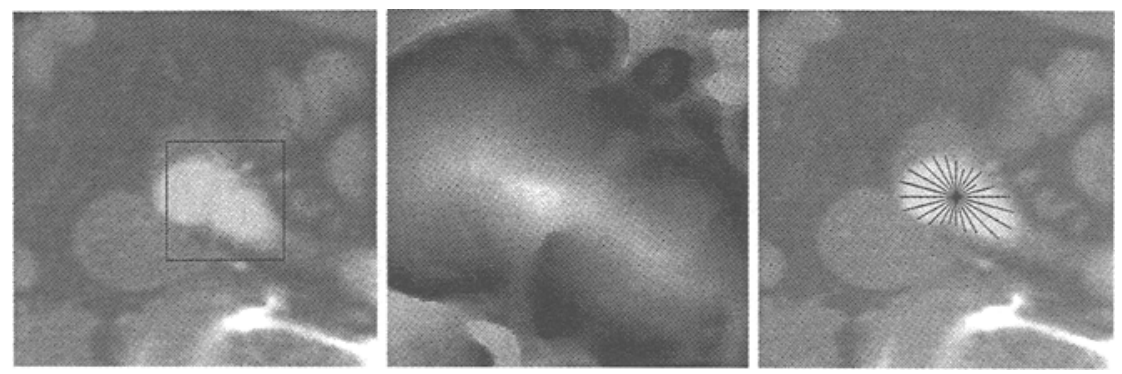

Fig. 4. An example of a bifurcation (left), and its confidence level image (middle), showing the largest peak in the middle. The right image displays the rays used, $\sigma=$ $3, t=4, N=12$.

the middle image of figure 4, the confidence level corresponding to the square in the left figure, is shown. This figure shows three definite peaks, with the largest in the middle. In the right image, the different rays are superimposed over the original image.

Intensity, scale and rotational invariance: the confidence level is invariant to the absolute value of the intensities in the lumen and to its size. However, several criteria have to be met, in order for the method to give proper results. Because of the thresholding of the gradient, the lumen itself should have a rather continuous grey level, and have higher intensity values than the surrounding tissue. The rotational invariance of the method depends on the number of lines that are used in computing the confidence level. The larger the number of lines, the better it approximates rotational invariant behaviour.

Outliers: if one of the rays 'misses' the border of the lumen, and stops at the edge of another more remote structure the contribution to the confidence level is almost zero. This is also the case if one of the rays accidentally enters one of the joining vessels, like the renal arteries as shown in the rightmost image from figure 1.

Interactivity: because of the normalization, the confidence level $C L$ is always within the range $[0,1]$. The confidence level can therefore be used to determine whether the tracking process went outside the artery, by inspecting its value or change in value during the tracking process.

Indication of the lumen borders: the confidence level is supposed to give a high value in the vicinity of the lumen center. Furthermore, the positions where the casting of the ray is terminated, give an indication of the lumen 
border. This information can be used as an initialization for more sophisticated segmentation methods, like active contour models [10]. In the quantification process, the minimum length of the lines $\left(d_{\min }\right)$ used in determining the lumen center, is stored. This measure is not very robust to noise and irregularities in the image, but is used to give a quick overview of the vessel morphology.

\subsection{Presentation of the results}

When the method is applied to a CTA dataset, the results are presented in several ways. The central lumen line is displayed on the EasyVision workstation during the tracking process. The quantitative results are presented in a 'click able' graph, displaying the minimum diameter $d_{\min }$ and the confidence level $C L$ at every cut plane, as shown in figure 5 . The graph can be used to interactively

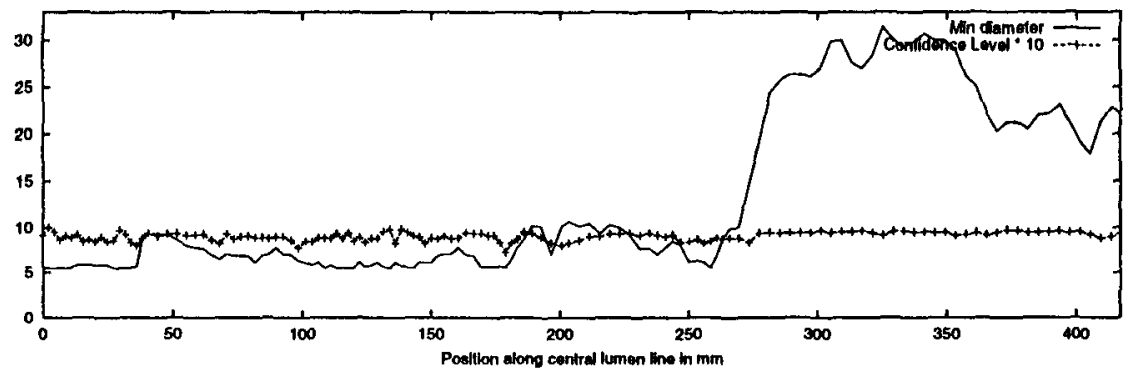

Fig. 5. Minimimum diameter in $\mathrm{mm}$. and confidence level, $\sigma=3, t=4, N=24$.

inspect the lumen cross sections. Pressing a position in the graph generates the corresponding cut plane along the computed central lumen line. Possible problem areas can thus be inspected.

During the tracking process, the end positions of the rays in every perpendicular plane are stored. Connecting these points to polygons yields a 3D representation of the computed lumen borders as shown in figure 6 . This $3 \mathrm{D}$ representation can be interactively manipulated to get an overview of the tortuosity of the iliac trajectory, and the neck of the aneurysm.

\section{Experiments and results}

The method was applied to 15 patients. For every patient, a starting position was manually determined in both left and right iliac arteries. The CTA acquired for the evaluation of candidates for the endovascular procedure, typically consist of 183 slices of $512 \times 512$ pixels, with a pixel size of $0.49 \mathrm{~mm}$, and an effective slice thickness of $2.0 \mathrm{~mm}$. A total of $140 \mathrm{cc}$ of contrast fluid is given intravenously with an injection rate of $3 \mathrm{cc} / \mathrm{sec}$. Scanning is performed at $140 \mathrm{KV} / 225 \mathrm{~mA}$., starting 30 seconds after onset of injection.

Based on experimental evidence, we selected default values for the free parameters $t, \sigma$ and $N$, which remained fixed for all clinical studies. 

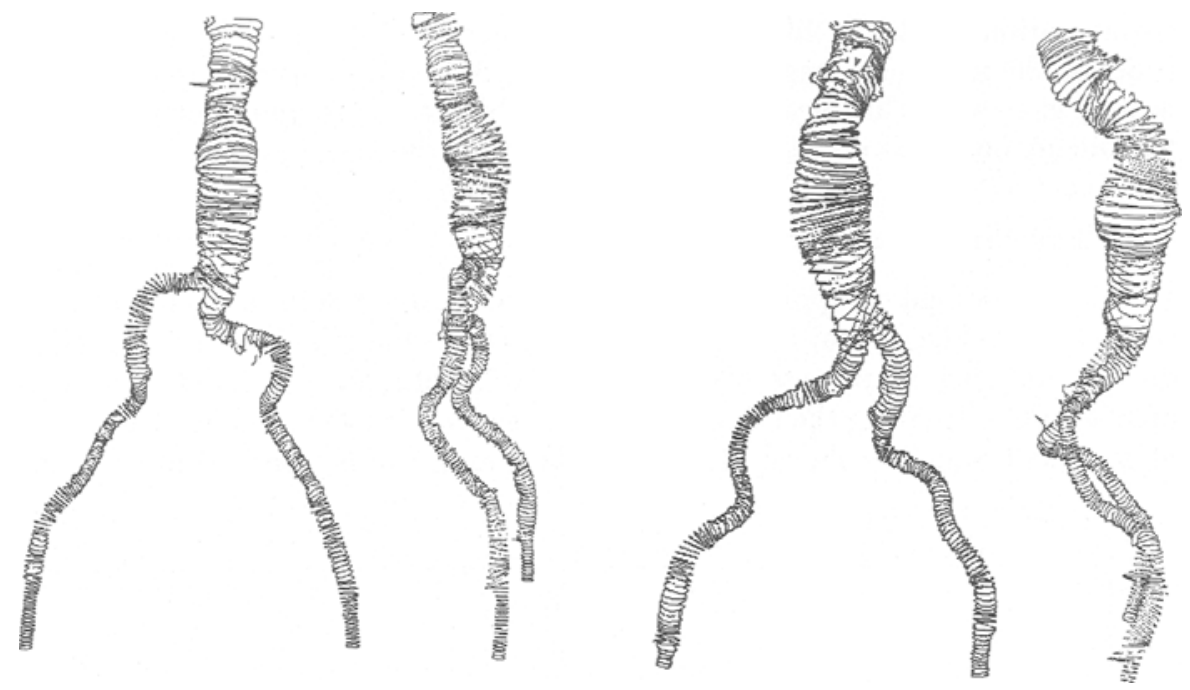

Fig. 6. 3D representations of the computed lumen borders from two patients, $\sigma=$ $3, t=4, N=24$.

The method was able to track both arteries to the top of the datasets for 14 out of 15 patients. The reason for the one failure was the low contrast in that specific dataset. The method accidentally detected the border of the thrombus, instead of the border of the lumen. The iliac arteries were correctly tracked in all the patients.

The average time for performing 150 iterations, including the computation of the $d_{\min }$ and the storage of the endpoints of the rays was less than one minute, on a SUN Sparc 5, $170 \mathrm{MHz}$.

\section{Conclusions and future work}

A method has been presented that is able to delineate an abdominal aorta aneurysm in less than one minute. The method is robust to typical problems occurring in 3D CTA data such as branching vessels, calcifications and shape variations. User initialization is minimal, and the clinician is able to supervise the tracking procedure interactively. The results are displayed in order to access problem areas quickly.

Initial experiments showed that in 14 out of 15 datasets, both vessels were completely tracked. Even in the highly curved iliac arteries the method was able to generate a central lumen line.

Currently, the results with respect to the minimum diameter are being validated with manually determined measurements. Also the method will be compared with manually determined central lumen lines.

The computed central lumen line and the estimated lumen borders will be used as an initialization for more advanced segmentation procedures. 


\section{Acknowledgments}

We thank Dr Ivo Broeders from the Department of Vascular Surgery of our hospital for providing us the CTA datasets. Paul Desmedt from Philips Medical Systems Best is acknowledged for his holp in building the prototype within the EasyScil reformat facility. This research is done within the context of the EASI project "European Applications for Surgical Interventions", subsidized by the European Commission under contract HC1012 in their "4th Framework Telematics Applications for Health" RTD programme.

\section{References}

1. I. A. J. M. Broeders, J. D. Blankensteijn, M. Olree, W. P. Th. M. Mali, and B. C. Eikelboom. Preoperative sizing of grafts for transfemoral endovascular aneurysm management: a prospective comparative study of spiral CT angiography, arteriography, and conventional CT imaging. Journal of Endovascular Surgery, 4:252-261, 1997.

2. C. Lorenz, I. C Carlsen, T. M. Buzug, C. Fassnacht, and J. Weese. Multi-scale line segmentation with automatic estimation of width, contrast and tangential direction in 2D and 3D medical images. In Proc. CVRMed and MRCAS 1997, number 1205 in Lecture notes in Computer Science, pages 233-242. Springer Verlag, Berlin, 1997.

3. V. Prinet and O. Monga. Vessel representation in 2D and 3D angiograms. In H. U. Lemke, M. W. Vannier, and K. Inamura, editors, Computer Assisted Radiology and Surgery, pages 240-245. Elsevier Publishers, Amsterdam, 1997.

4. Th. M. Koller, G. Gerig, G. Székely, and D. Dettwiler. Multiscale detection of curvilinear structures in 2D and 3D image data. In Fifth International Conference on Computer Vision, pages 864-869, 1995.

5. A. B. Houtsmuller, A. W. M. Smeulders, H. T. M. van der Voort, J. L. Oud, and N. Nanninga. The homing cursor: A tool for three-dimensional chromosome analysis. Cytometry, 14:501-509, 1993.

6. T. O'Donnel, A. Gupta, and T. Boult. A new model for the recovery of cylindrical structures from medical image data. In Proc. CVRMed and MRCAS 1997, number 1205 in Lecture notes in Computer Science, pages 223-232. Springer Verlag, Berlin, 1997.

7. V. Juhan, B. Nazarian, K. Malkani, R. Bulot, J. M. Bartoli, and J. Sequeira. Geometrical modelling of abdominal aortic aneurysms. In Proc. CVRMed and MRCAS 1997, number 1205 in Lecture notes in Computer Science, pages 243-252. Springer Verlag, Berlin, 1997.

8. J. Beier, R. Siekmann, F. Müller, J. Tröger, H. Schedel, G. Biamino, E. Fleck, and R. Felix. Planning and control of aortic stent implantations using 3D reconstructions based on computer tomography. In H. U. Lemke, M. W. Vannier, and K. Inamura, editors, Computer Assisted Radiology, pages 716-720. Elsevier Publishers, Amsterdam, 1996.

9. M. Fiebich, M. Tomiak Mitchell, R. M. Engelmann, and K. R. Hoffmann. Automatic segmentation in CT angiography of the abdominal aorta. In H. U. Lemke, M. W. Vannier, and K. Inamura, editors, Computer Assisted Radiology and Surgery, pages 277-282. Elsevier Publishers, Amsterdam, 1997.

10. M. Kass, A. P. Witkin, and D. Terzopoulos. Snakes: Active contour models. International Journal of Computer Vision, 1:321-331, 1988. 